\title{
¿Hay beneficios psicosociales por la práctica Pilates? Un análisis de la literatura científica
}

\author{
Are there psychosocial benefits for Pilates practice? \\ An analysis of the scientific literature
}

\section{Existem benefícios psicossociais para a prática de Pilates? Uma análise da literatura científica}

\author{
Salvador Boix Vilella ${ }^{1}$, Eva León Zarceño ${ }^{2}$ y Miguel Ángel Serrano Rosa ${ }^{3}$ \\ ${ }^{1}$ Departamento de Orientación, Instituto de Educación Secundaria Antonio Serna, Albatera, ${ }^{2}$ Departamento de Psicología de la Salud, Universidad Miguel \\ Hernández de Elche, ${ }^{3}$ Departamento de Psicobiologia, Universitat de València.
}

Resumen: En los últimos 5 años, las bases de datos analizadas muestran un notable incremento en el número de publicaciones sobre Pilates. La enorme difusión y popularidad del método Pilates podría deberse a los beneficios físicos, psíquicos y sociales que las personas podrían obtener con su práctica. Desde el ámbito científico, existe la necesidad de actualizar y evaluar objetivamente los resultados ya existentes sobre los posibles beneficios que puede producir la práctica de Pilates sobre la salud. Esta revisión, pretende comprobar si hay una suficiente fundamentación científica que justifique sus beneficios en la salud psicológica de los practicantes de Pilates. Para ello se ha realizado una búsqueda sistemática, utilizando como criterio de búsqueda la palabra "Pilates", en las bases de datos: ScienceDirect, Pubmed, Lilacs, PsycARTICLES, CSIC-ISOC y Dialnet. Entre las variables psicológicas que han sido estudiadas las más relevantes han resultado ser la calidad de vida, la depresión, el estado de ánimo, la autoeficacia generalizada y los aspectos relativos al sueńo. Por otro lado, teniendo en cuenta las singularidades del propio método se van a exponer futuras líneas de investigación. Palabras clave: Ejercicio, método Pilates, salud mental, psicología social, salud laboral.

Abstract: In the last 5 years, the analyzed databases show a remarkable increase in the number of publications on Pilates. The enormous popularity of Pilates may be due to physical and psychological benefits that people could get to their practice. From the scientific field, there is a need to evaluate objectively the existing results on the potential benefits of the Pilates Method. So this work is to check whether there is a sufficient scientific basis to justify their psychological health benefits practitioners. For this we have performed a systematic search, using as search criteria the word "Pilates" in databases: ScienceDirect, Pubmed, Lilacs, PsycARTICLES, CSIC-ISOC and Dialnet. Among the psychological variables that have been studied the most relevant have proven the quality of life, depression, mood, generalized self-efficacy and aspects of sleep. Also taking into account the peculiarities of the method itself will narrow future research.

Keywords: Exercise, Pilates method, mental health, social psychology, occupational health.

Resumo: Nos últimos 5 anos, os bancos de dados analisados mostram um aumento notável no número de publicaçōes sobre Pilates. A enorme difusão e popularidade do Pilates pode ser devido aos benefícios físicos, mentais e sociais que as pessoas pudem começar a sentir com a sua prática. No campo científico, há uma necessidade de atualizar, objetivar e avaliar os resultados existentes sobre os potenciais benefícos que podem produzir a prática de Pilates na saúde. Esta revisão visa estabelecer a existencia da base científica, que seja suficiente para justificar seus benefícios psicológicos de saúde desta prática. Para isso, foi realizada uma busca sistemática, utilizando como critério de busca a palavra "Pilates" em bancos de dados: ScienceDirect, Pubmed, Lilacs, PsycARTICLES, CSIC-ISOC e Dialnet. Entre as variáveis psicológicas que têm sido estudados os mais relevantes têm comprovado a qualidade de vida, depressão, humor, generalizada auto-eficácia e os aspectos do sono. Tendo em vista as peculiaridades do método em si vai diminuir as dúvidas referentes a este assunto.

Palavras chave: Exercício, Pilates, saúde mental, psicologia social, saúde ocupacional.

\section{Introducción}

Joseph Hubertus Pilates creó un sistema de ejercicios al que llamó contrología. Concepto que él mismo definió en su libro como: "la ciencia y arte del desarrollo coordinado de la mente, el cuerpo y el espíritu, a través de movimientos naturales, bajo el estricto control de la voluntad" (Pilates y Miller, 1945).

Dirección para correspondencia [Correspodence address]: Miguel Ángel Serrano Rosa, Departamento de Psicobiología, Facultad de Psicología, Universitat de València. Avenida Blasco Ibáńez, 21, 46010 Valencia (España).E-mail: m.angel.serrano@uv.es
Según Fernández, Santana y Merino (2011), Joseph Pilates fue un auténtico pionero o adelantado en su tiempo (casi un siglo a las tendencias actuales) al ser de los primeros occidentales en estudiar diferentes disciplinas orientales como el yoga o el zen. De las concepciones orientales recoge en su método, características como la interiorización de uno mismo, la adaptación a los ritmos naturales para conseguir equilibrio interior, el control de los gestos y la concepción global de la persona que se presenta como una unidad cuerpo-mente- 
espíritu (Isacowitz, 2009; Siler, 2000; Winsor, 2002). De las actividades occidentales practicadas durante su infancia (boxeo, esquí, danza y gimnasia) obtiene la importancia de la fuerza, la resistencia y la intensidad del ejercicio (Levine, Kaplanek, Scafura y Jaffe, 2007).

El Método Pilates se compone de más de 500 ejercicios. Éstos están basados en repeticiones breves acompañadas del acompasamiento de la respiración con el fin de acondicionar el cuerpo (Ramos, 2007). Existen, al menos, dos modalidades de Pilates. La primera modalidad conocida como Pilates Mat, donde se lleva a cabo la técnica sólo en una colchoneta, utilizando como resistencia el peso del propio cuerpo. La segunda mediante máquinas que se realiza usando aparatos desarrollados por el propio Pilates que han llegado hasta nuestros días, aunque con las correspondientes adaptaciones (Latey, 2001).

Actualmente se está convirtiendo en uno de los sistemas de ejercicio más demandados desde diversos campos: rehabilitación, acondicionamiento general, complemento para deportes específicos, embarazadas, personas mayores, niños, etc. (González-Gálvez y Sainz de Baranda, 2011). Algunos autores consideran que el Método Pilates mejora la condición física (fuerza, flexibilidad, coordinación y equilibrio), aumenta la conciencia corporal, reeduca las actitudes posturales, aumenta la creatividad, la memoria, la autoestima, reduce el estrés, el cansancio, la agresividad, el dolor, mejora el estado de ánimo, la densidad ósea, la postura, etc. (Adamany y Loigerot, 2006; Anderson, 2010; Anderson y Spector, 2000; Muirhead, 2004; Pilates y Miller, 1945; Shedden y Kravitz, 2006). La práctica habitual de actividad física durante el tiempo libre influye positivamente sobre el bienestar psicológico y emocional de las personas (Deslandes, Moraes, Ferreira, Veiga, Silveira, Mouta, Pompeu, Coutinho y Laks, 2009). Un estilo de vida activo ayuda a mantener las funciones cognitivas en edades avanzadas (Flicker, 2009) y mejora la calidad de vida del practicante (Sjögren, Nissinem, Järvenpää, Ojanen, Vanharanta y Mälkiä, 2006).

Dentro del marco laboral, la utilización de programas de actividad física en las diferentes organizaciones puede ser una medida preventiva para mejorar la salud psicológica percibida de los trabajadores (Serrano y Boix, 2012) ya que se ha demostrado que todo lo que rodea al trabajo remunerado acaba por tener un impacto sustancial en la salud mental (Warr, 2003). Para contrarrestar los posibles efectos negativos del trabajo, la salud laboral se encarga de aquellos estudios o acciones que tienen como finalidad conocer la importancia del trabajo en las alteraciones de la salud en una población, así como establecer medidas preventivas (López Menchero, 2009).

A pesar del elevado número de practicantes y de la gran cantidad de beneficios que se le atribuyen al Método Pilates no existe aún una evidencia científica sólida sobre sus efectos (Fernández et al., 2011; González-Gálvez, Sainz de Baranda, García-Pastor y Aznar, 2012). Si a ello le añadimos que, en los últimos años, se ha producido un aumento notable de publicaciones científicas sobre Pilates, nos hace plantearnos la necesidad de realizar un análisis de la literatura científica (Fernández-Ríos y Buela, 2009; Perestelo, 2013; Sánchez-Meca y Botella, 2010) para conocer el estado actual de la cuestión.

Por todo ello, los objetivos de nuestro trabajo son: (a) tratar de resumir y analizar las publicaciones sobre los efectos del método Pilates sobre la salud psicosocial y salud laboral, con el fin de identificar los aspectos psicosociales y laborales que se ven afectados por esta actividad física; (b) sugerir líneas de actuación para futuras investigaciones.

\section{Método}

\section{Estrategia de búsqueda bibliográfica}

Las bases de datos seleccionadas para localizar los trabajos científicos han sido: ScienceDirect, Pubmed, Lilacs, PsycARTICLES, CSIC-ISOC y Dialnet. De esta forma podemos acceder a las principales publicaciones existentes y revisar si se obtienen beneficios sobre la salud psicológica, a través de la práctica del Método Pilates.

Como criterio de búsqueda se utilizó la palabra "Pilates" que siempre estuvo presente en uno de los campos de búsqueda. El resto de campos estuvieron subordinados con el operador booleano "and" y completados por una de los siguientes descriptores en inglés: health psychological, health social, health psychosocial, depression, stress, anxiety, occupational health, burnout, self-esteem, emotional stability, engagement, optimism y job satisfaction.

\section{Criterios de inclusión y exclusión}

En nuestro trabajo de revisión se incluyen personas de todas las edades y con cualquier nivel de condición física. Se seleccionaron y revisaron los artículos científicos publicados hasta el final de 2013. La selección de los estudios científicos estuvo basada en los siguientes criterios de inclusión: (a) estudios de revisión; (b) estudios descriptivos; (c) estudios de intervención; (d) utilizar el Método Pilates como variable diferenciadora; (e) evaluar los efectos del método Pilates sobre alguna variable psicológica.

Por otro lado, se excluyeron del análisis los siguientes casos: (a) estudios científicos publicados en forma de resumen y/o comunicaciones cortas; (b) capítulos de libros; (c) trabajos redactados en idioma distinto al inglés, español o portugués; (d) artículos que engloban ejercicios de Pilates junto a otras actividades físico-deportivas en un mismo grupo. De esta forma se intenta evitar posibles distorsiones en los resultados finales ya que no podríamos asegurar que el Pilates es la única actividad que genera los posibles beneficios encontrados. 


\section{Resultados}

La consulta en las bases de datos aporta la información necesaria para desarrollar la revisión. En la Tabla 1 se muestra el número total de trabajos encontrados en función de las palabras claves delimitadas anteriormente. En el caso de
Dialnet, sólo es posible realizar la búsqueda con utilizando como descriptor "Pilates". También se ha querido constatar el aumento progresivo de publicaciones que se está produciendo alrededor al Método Pilates (véase Figura 1) otorgándole un mayor peso específico e importancia dentro de la comunidad científica.

Tabla 1. Resultados de la búsqueda bibliográfica según las palabras clave y el número de artículos encontrados

\begin{tabular}{lcccccc}
\hline & Science Direct & Pubmed & Lilacs & CSIC-ISOC & PsycARTICLES & Dialnet \\
\hline Pilates & 769 & 124 & 182 & 4 & 1 & 65 \\
Pilates AND Health Psychological & 242 & 5 & 7 & 0 & 1 & - \\
Pilates AND Health Social & 323 & 3 & 1 & 0 & 0 & - \\
Pilates AND Health Psychosocial & 132 & 2 & 16 & 0 & 0 & - \\
Pilates AND Depression & 212 & 3 & 5 & 0 & 0 & - \\
Pilates AND stress & 338 & 2 & 4 & 0 & 0 & - \\
Pilates AND Anxiety & 197 & 1 & 1 & 0 & 0 & - \\
Pilates AND Occupational Health & 141 & 1 & 1 & 0 & 0 & - \\
Pilates AND Burnout & 16 & 0 & 0 & 0 & 0 & - \\
Pilates AND Self-esteem & 1 & 4 & 0 & 0 & 0 & - \\
Pilates AND Emotional Stability & 74 & 0 & 0 & 0 & 0 & - \\
Pilates AND Engagement & 63 & 0 & 0 & 0 & 0 & - \\
Pilates AND Optimism & 15 & 0 & 0 & 0 & 0 & - \\
Pilates AND Job Satisfaction & 36 & 0 & 0 & 0 & 0 & - \\
\hline
\end{tabular}

Figura 1. Número de publicaciones con la palabra clave "Pilates" a lo largo de los ańos.

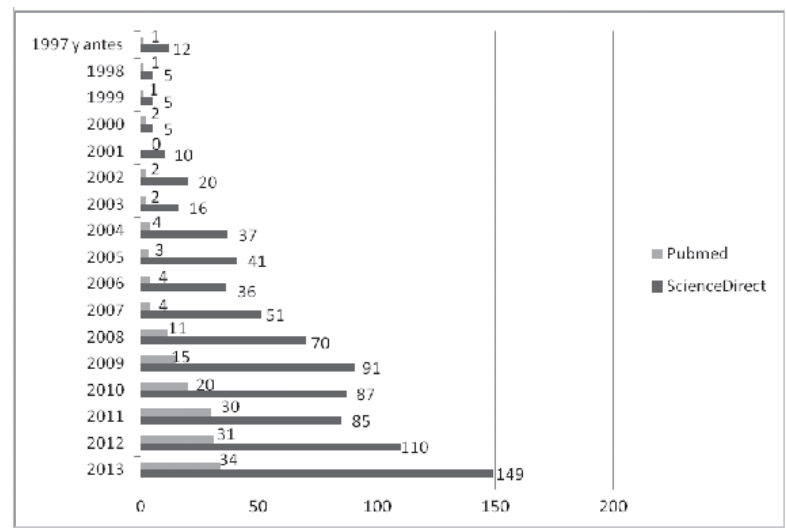

De todas las publicaciones encontradas en la Tabla 1, 28 trabajos cumplieron con los criterios de selección establecidos. A pesar de ello, 6 publicaciones fueron excluidas de la revisión, en el último momento, por presentar limitaciones en el apartado metodológico. La primera limitación que motivó la exclusión de 3 artículos (Anderson, 2005; Bian, Sun, Lu, Yao, Chen y Li, 2013; Keays, Harris, Lucyshyn y MacIntyre,
2008) tuvo que ver con el reducido tamaño de las muestras que hacía imposible sacar conclusiones. Los otros 3 artículos (McGrath, O'Malley y Hendrix, 2011; Mendonça, Terreri, Silva, Neto, Pinto, Natour y Len, 2013; Vieira, Faria, Wittmann, Teixeira y Nogueira, 2013) también fueron eliminados por no definir con la suficiente claridad el método Pilates utilizado. Finalmente, 22 trabajos han sido codificados en la Tabla 2. En ella se muestra: autor, autora o autores, ańo de publicación, diseño empleado, número de sujetos, variables estudiadas, intervención y resultados más destacados. La mayoría de los trabajos que se muestran en ella no se han centrado exclusivamente en aspectos psicológicos (Altan, et al., 2009; Borges et al., 2013; Cruz-Ferreira et al., 2011; Eyigor et al., 2010; Lange et al.,2000; Mokhtari et al., 2012; Priebe et al., 2013; Segal et al., 2004; Siqueira et al., 2010). Es por ello, que se han podido omitir aquellos otros resultados del trabajo que no se relacionan con los objetivos marcados en nuestra revisión. 
Tabla 2. Descripción de los estudios incluidos en la revisión

\begin{tabular}{|c|c|c|c|c|}
\hline Estudio & Diseño & Sujetos & Variables/intervención & Resultados \\
\hline $\begin{array}{l}\text { Lange et al., } \\
(2000)\end{array}$ & Revisión & $\begin{array}{l}\text { Publicaciones so- } \\
\text { bre los beneficios } \\
\text { y el aprendizaje } \\
\text { de Pilates }\end{array}$ & $\begin{array}{l}\text { Estado de salud percibida, humor, estado } \\
\text { motivacional, disfrute de la vida, energía y } \\
\text { entusiasmo. } \\
\text { Incluye cualquier tipo de Pilates. }\end{array}$ & $\begin{array}{l}\text { El funcionamiento psicológico es una } \\
\text { de las categorías revisadas, aunque se } \\
\text { concluye que no existen publicaciones } \\
\text { científicas suficientes. }\end{array}$ \\
\hline $\begin{array}{l}\text { Segal et al, } \\
(2004)\end{array}$ & $\begin{array}{l}\text { Estudio } \\
\text { prospectivo } \\
\text { observacional }\end{array}$ & $\begin{array}{l}\text { Pilates } \mathrm{N}=47 \\
\text { Cumplieron cri- } \\
\text { terio de selección } \\
\mathrm{n}=32(31 \text { mujeres } \\
\text { y } 1 \text { hombre })\end{array}$ & $\begin{array}{l}\text { Estado de salud (SBIA). } \\
\text { Criterio inclusión: no faltar a ninguna se- } \\
\text { sión. Se realiza una entrevista previa donde } \\
\text { dan su consentimiento. } \\
\text { Mat pilates: } 1 \text { sesión de } 60 \text { ' x semana } \\
\text { durante } 6 \text { meses. } \\
\text { Mediciones al inicio, } 2 \text {, } 4 \text { y } 6 \text { meses. }\end{array}$ & $\begin{array}{l}\text { Mejoras sin cambios significativos en el } \\
\text { estado de salud. Se considera que no es } \\
\text { fácil establecer efectos del Pilates sobre } \\
\text { el estado de salud. }\end{array}$ \\
\hline $\begin{array}{l}\text { Bernardo et } \\
\text { al., (2007) }\end{array}$ & Revisión & $\begin{array}{l}\text { Publicaciones } \\
\text { Pilates } \mathrm{N}=277 \\
\text { Incluidas } \mathrm{n}=3\end{array}$ & $\begin{array}{l}\text { Revisa cualquier tipo de Pilates en adultos } \\
\text { sanos y propone futuras líneas de trabajo. }\end{array}$ & $\begin{array}{l}\text { El estado de salud es la única variable } \\
\text { psicosocial citada en la revisión (Segal } \\
\text { et al., 2004). Llegan a la conclusión que } \\
\text { se necesitan estudios experimentales, } \\
\text { con sujetos asignados al azar, con una } \\
\text { tamańo de muestra grande y un mode- } \\
\text { lo de Pilates bien definido. }\end{array}$ \\
\hline $\begin{array}{l}\text { Ekici et al., } \\
(2008)\end{array}$ & $\begin{array}{l}\text { Estudio } \\
\text { aleatorizado } \\
\text { controlado }\end{array}$ & $\begin{array}{l}\text { Pacientes fibro- } \\
\text { mialgia } N=51 \\
\text { Pilates } n=27 \\
\text { Grupo masaje } \\
\mathrm{n}=24\end{array}$ & $\begin{array}{l}\text { Depresión (BDI). } \\
\text { Pilates: } \\
3 \text { veces x semana durante } 4 \text { semanas. }\end{array}$ & $\begin{array}{l}\text { Mejoras significativas se determinaron } \\
\text { en ambos grupos. Estos dos enfoques } \\
\text { pueden ser utilizados para disminuir los } \\
\text { síntomas de depresión en mujeres con } \\
\text { fibromialgia. }\end{array}$ \\
\hline $\begin{array}{l}\text { Altan et al., } \\
(2009)\end{array}$ & $\begin{array}{l}\text { Estudio } \\
\text { clínico } \\
\text { aleatorizado, } \\
\text { prospectivo, } \\
\text { controlado } \\
\text { doble ciego }\end{array}$ & $\begin{array}{l}\text { Mujeres con fibro- } \\
\text { mialgia } \mathrm{N}=50 \\
\text { Pilates } \mathrm{n}=25 \\
\text { Grupo control } \\
\mathrm{n}=25 \text { ejercicios } \\
\text { hogar. }\end{array}$ & $\begin{array}{l}\text { Calidad de vida (FIQ). } \\
\text { Pilates } 3 \text { clases de } 60 \text { x semana durante } 12 \\
\text { semanas. } \\
\text { Pre-post test (semana } 12 \text { y } 24 \text { ). }\end{array}$ & $\begin{array}{l}\text { Mejora significativa en semana } 12 \text { y } \\
24 \text { en el Fibromialgia Impact Quest. } \\
\text { Aunque se apunta que mejora la calidad } \\
\text { de vida, al ser el primer estudio clínico } \\
\text { en este campo se recomiendan más tra- } \\
\text { bajos para evaluar su valor terapéutico. }\end{array}$ \\
\hline $\begin{array}{l}\text { Caldwell et } \\
\text { al., (2009) }\end{array}$ & $\begin{array}{l}\text { Estudio } \\
\text { controlado } \\
\text { comparativo }\end{array}$ & $\begin{array}{l}\text { Edad universita- } \\
\text { ria } N=127 \\
\text { Pilates } n=51 \\
\text { Taichí } n=35 \\
\text { Recreación } n=41\end{array}$ & $\begin{array}{l}\text { Percepción de autoeficacia (SRE), calidad } \\
\text { percibida del sueńo (PSQI) y estado de } \\
\text { ánimo (FDMS). } \\
\text { Mat Pilates: } \\
2 \text { clases de } 75 \text { ' x semana durante } 6 \text { meses o } \\
3 \text { clases de } 50 \text { 'x semana durante } 6 \text { meses. }\end{array}$ & $\begin{array}{l}\text { El humor mejoró en el grupo de Pilates } \\
\text { significativamente. También aumentó } \\
\text { la autoeficacia y había una tendencia a } \\
\text { la mejora en la calidad del sueńo. }\end{array}$ \\
\hline $\begin{array}{l}\text { Caldwell et } \\
\text { al., (2010) }\end{array}$ & Experimental & $\begin{array}{l}\text { Universitarios } \\
N=166 \\
\text { Pilates } \mathrm{n}=80 \\
\text { Girokinesis } \mathrm{n}=48 \\
\text { Taichí } \mathrm{n}=38\end{array}$ & $\begin{array}{l}\text { Autoeficacia (SRE), estado de ánimo } \\
\text { (FDMS), estrés percibido (PSS4), atención } \\
\text { (FFMQ) y calidad del sueño (PSQI). Un } \\
\text { total de } 15 \text { clases de Mat Pilates, con la po- } \\
\text { sibilidad de hacer: } 2 \text { clases de } 75 \text { ' x semana } \\
\text { ó } 3 \text { clases de 50’ x semana. }\end{array}$ & $\begin{array}{l}\text { Tras las mediciones al inicio, mitad y } \\
\text { final del trabajo se concluye que el Mé- } \\
\text { todo Pilates puede aumentar la atención, } \\
\text { el estado de ánimo y el estrés percibido, } \\
\text { que explican, en parte, la mejora de la } \\
\text { calidad del sueńo. }\end{array}$ \\
\hline $\begin{array}{l}\text { Siqueira et } \\
\text { al., (2010) }\end{array}$ & Experimental & $\begin{array}{l}\text { Mujeres mayores } \\
\mathrm{N}=52 \\
\text { Pilates } \mathrm{n}=27 \\
\text { Grupo control } \\
\mathrm{n}=25\end{array}$ & $\begin{array}{l}\text { Autonomía (Protocolo GDLAM) y calidad } \\
\text { de vida (WHOQOL-OLD). } \\
\text { Pilates máquinas: } 2 \text { clases de } 60 \text { x x semana } \\
\text { durante } 8 \text { semanas. El Grupo control no } \\
\text { hace ejercicio. Mediciones pre-post test. }\end{array}$ & $\begin{array}{l}\text { El grupo Pilates mejora la autonomía } \\
\text { personal y la calidad del índice de } \\
\text { vida. En el grupo control en cambio no } \\
\text { existen diferencias significativas. }\end{array}$ \\
\hline $\begin{array}{l}\text { Eyigor et al., } \\
(2010)\end{array}$ & $\begin{array}{l}\text { Estudio } \\
\text { controlado } \\
\text { aleatorizado }\end{array}$ & $\begin{array}{l}\text { Pacientes cáncer } \\
\text { de mama } \mathrm{n}=52 \\
\text { Divididos en } \\
\text { grupo control y } \\
\text { Pilates }\end{array}$ & $\begin{array}{l}\text { Depresión (BDI) y la calidad de vida } \\
\text { (GLQ-30). } \\
\text { Mat Pilates } 3 \text { clases de } 60 \text { ' x semana duran- } \\
\text { te } 8 \text { semanas. }\end{array}$ & $\begin{array}{l}\text { No se observaron mejoras en el grupo } \\
\text { Pilates en el índice de depresión ni en } \\
\text { calidad de vida. El Pilates es eficaz y } \\
\text { seguro en el cáncer de mama, pero se } \\
\text { necesitan más estudios. }\end{array}$ \\
\hline
\end{tabular}




\begin{tabular}{|c|c|c|c|}
\hline Estudio & Diseño & Sujetos & Variables/intervención \\
\hline $\begin{array}{l}\text { Tinoco, M. } \\
\text { y Jiménez, } \\
\text { M. (2010) }\end{array}$ & Revisión & $\begin{array}{l}\text { Publicaciones } \\
\mathrm{n}=277\end{array}$ & $\begin{array}{l}\text { Evaluar investigaciones sobre Pilates y pro- } \\
\text { poner futuras líneas de investigación. }\end{array}$ \\
\hline $\begin{array}{l}\text { Cruz-Fe- } \\
\text { rreira et al., } \\
(2011)\end{array}$ & Revisión & $\begin{array}{l}\text { Artículos inclui- } \\
\text { dos } n=17\end{array}$ & $\begin{array}{l}\text { Efectividad del Método Pilates en personas } \\
\text { sanas. }\end{array}$ \\
\hline
\end{tabular}

(2011)

\begin{tabular}{lll}
\hline Cruz-Fe- & Ensayo & Mujeres $\mathrm{N}=62$ \\
rreira et al., & controlado & Pilates $\mathrm{n}=38$ \\
$(2011)$ & aleatorio & Grupo control \\
& & $\mathrm{n}=24$
\end{tabular}

\author{
Satisfacción con la vida (SWLS), auto- \\ concepto físico (CAF) y estado de salud \\ (EQ-5D). \\ Pilates Mat 2 sesiones de 60' x semana \\ durante 6 meses. \\ Mediciones: inicio, 3 y 6 meses.
}

Resultados

En el apartado psicosocial hay mejoras sin cambios significativos en: el estado de salud percibida, en el estado de salud emocional y en la calidad del sueño. Sí hay cambios significativos en: autoeficacia y humor.

Evidencia limitada: satisfacción con la vida, autoconcepto y percepción del estado de salud. No hay evidencia: autoeficacia, estado ánimo, calidad del sueño, equilibrio mental, calidad de vida y autonomía.

Pilates mostró mejoras significativas (pre-post y half-post test) en: satisfacción con la vida, la percepción de la apreciación de personas, la percepción de apariencia física y percepción de funcionalidad. También el estado de salud (pre-post test) el autoconcepto físico y la percepción de funcionalidad (half test-post test).

\begin{tabular}{lll}
\hline Altan et al., & Estudio & Pacientes con \\
aleatorizado, & epicondilitis an- \\
& prospectivo, & quilosante $\mathrm{N}=55$ \\
controlado, & Pilates $\mathrm{n}=30$ \\
ensayo ciego & Grupo control \\
simple & $\mathrm{n}=25$
\end{tabular}

Estado funcional (FIQ) y Calidad de vida (NHP).

Grupo Pilates siguiendo sus principios básicos consta 9 módulos de media o baja dificultad.

3 veces 60' x semana durante 12 semanas. Grupo control: programas de tratamiento estándar anteriores.

\begin{tabular}{|c|c|c|c|}
\hline $\begin{array}{l}\text { Stan et al., } \\
(2012)\end{array}$ & Revisión & $\begin{array}{l}\text { Artículos } \mathrm{N}=3 \\
\text { Incluidos } \mathrm{n}=2\end{array}$ & $\begin{array}{l}\text { Sueño, fatiga, ansiedad, depresión, angustia } \\
\text { y calidad de vida. "Pilates and breast } \\
\text { cancer". }\end{array}$ \\
\hline
\end{tabular}

\begin{tabular}{lll}
\hline $\begin{array}{l}\text { González- } \\
\text { Gálvez et al., Revisión }\end{array}$ & $\begin{array}{l}\text { Publicaciones } \\
\mathrm{n}=70\end{array}$ & $\begin{array}{l}\text { La metodología de intervención más } \\
\text { habitual al estudiar Pilates, emplea: 11-20 } \\
(2012)\end{array}$ \\
& $\begin{array}{l}\text { sujetos, en sesiones de 60', } 2 \text { o 3 veces por } \\
\text { semana. El programa de intervención más } \\
\text { largo es de } 6 \text { meses y el rango más común } \\
\text { es 4-12 semanas. }\end{array}$ \\
\hline
\end{tabular}

Stan et al., Estudio de un Supervivientes de Calidad de vida (FACT-B), estado de (2012) solo brazo cáncer de mama ánimo (POMS) y la imagen corporal $\mathrm{N}=15 \quad$ (MBSRQ).

Completaron Mat Pilates: $3-5$ clases $45^{\prime}$ x semana duranestudio $n=13$ te 12 semanas. Mediciones pre-post test.

\begin{tabular}{llll}
\hline Leopoldino & Estudio clíni- & Voluntarios N=96 & Nivel de somnolencia (ESS) y calidad de \\
et al., (2013) & $\begin{array}{l}\text { co prospec- } \\
\text { Cumplen criterio }\end{array}$ & vida (SF-36). \\
& tivo & $\mathrm{n}=30$ & Criterio inclusión: no haber sido operado \\
& & & en últimos 6 meses. Pilates mat 2 veces x \\
& & semana durante 12 semanas. Mediciones: \\
& & pre-post test
\end{tabular}
Pilates muestra una mejoría significativa en el estado funcional. La comparación de los grupos mostró resultados significativamente superiores en el grupo Pilates. Se considera que es un método efectivo y seguro para pacientes con epicondilitis.

No existe, una fuerte evidencia para apoyar el pilates después del tratamiento del cáncer de mama debido a las escasas investigaciones.

De la revisión de los estudios científicos sobre Pilates sólo uno trata aspectos psicológicos (Altan, 2009). Los resultados muestran mejoras en la calidad de vida en pacientes con fibromialgia.

\footnotetext{
Mejoras significativas en: calidad de vida, estado de ánimo y en la imagen corporal. Las mejoras en los resultados psicológicos son prometedores y merecen mayor evaluación en un estudio aleatorizado controlado.

La población sedentaria que participó en este programa de Pilates de 12 semanas experimentaron mejoras en la calidad del sueño y la calidad de vida.
} 


\begin{tabular}{|c|c|c|c|c|}
\hline Estudio & Diseño & Sujetos & Variables/intervención & Resultados \\
\hline $\begin{array}{l}\text { Küçükçakır } \\
\text { et al., (2013) }\end{array}$ & $\begin{array}{l}\text { Estudio } \\
\text { aleatorizado } \\
\text { prospectivo, } \\
\text { controlado y } \\
\text { doble ciego. }\end{array}$ & $\begin{array}{l}\text { Mujeres con } \\
\text { osteoporosis } \\
\text { posmenopáusica } \\
\mathrm{N}=70 \\
\text { Divididos en gru- } \\
\text { po casa y Pilates }\end{array}$ & $\begin{array}{l}\text { Calidad de vida (SF-36). } \\
\text { Pilates: } 2 \text { veces x semana durante } 1 \text { año. } \\
\text { Grupo casa: ejercicios de extensión torácica. }\end{array}$ & $\begin{array}{l}\text { Una mejoría significativa se observó } \\
\text { en todos los parámetros de evaluación } \\
\text { al final del programa de ejercicio en el } \\
\text { grupo Pilates. El Pilates puede ser una } \\
\text { alternativa de tratamiento eficaz para } \\
\text { la calidad de vida en pacientes con } \\
\text { osteoporosis posmenopáusica. }\end{array}$ \\
\hline $\begin{array}{l}\text { Gámez- } \\
\text { Iruela, J. y } \\
\text { Sedeńo- } \\
\text { Vidal, A. } \\
\text { (2013) }\end{array}$ & Revisión & $\begin{array}{l}\text { Artículos } \mathrm{N}=22 \\
\text { Ensayos clínicos } \\
\text { aleatorizados } \\
\mathrm{n}=17 \\
\text { Revisiones } \mathrm{n}=4 \\
\text { Metaanálisis } \mathrm{n}=1\end{array}$ & Estrategia de búsqueda: "fibromialgia". & $\begin{array}{l}\text { Los tratamientos basados en el ejercicio } \\
\text { físico, liberación miofascial, terapia } \\
\text { craneosacra y Método Pilates desarro- } \\
\text { llan un aumento del bienestar y calidad } \\
\text { de vida de los pacientes a corto y medio } \\
\text { plazo. }\end{array}$ \\
\hline $\begin{array}{l}\text { Mokhtari et } \\
\text { al., (2013) }\end{array}$ & $\begin{array}{l}\text { Cuasi-experi- } \\
\text { mental } \\
\text { Pre-post }\end{array}$ & $\begin{array}{l}\text { Personas mayores } \\
\mathrm{n}=30\end{array}$ & $\begin{array}{l}\text { Depresión (GDS). } \\
\text { Las primeras } 6 \text { semanas Mat Pilates y las } \\
\text { otras con bandas. } 3 \text { sesiones de } 60^{\prime} \text { x sema- } \\
\text { na durante } 12 \text { semanas. }\end{array}$ & $\begin{array}{l}\text { Los ejercicios de Pilates disminuyen la } \\
\text { depresión en personas mayores (62-80 } \\
\text { años). }\end{array}$ \\
\hline $\begin{array}{l}\text { Borges et al., } \\
(2013)\end{array}$ & $\begin{array}{l}\text { Ensayo clíni- } \\
\text { co aleatoriza- } \\
\text { do, cruzado. }\end{array}$ & $\begin{array}{l}\text { Pacientes con } \\
\text { dolor lumbar } \\
\text { crónico } n=22 \\
\text { Grupo A } n=11 \\
\text { Grupo } B n=11\end{array}$ & $\begin{array}{l}\text { Calidad de vida (SF-36). } \\
\text { Grupo A: Pilates- control. Grupo B: } \\
\text { control-Pilates Combina Mat Pilates y } \\
\text { máquinas. } \\
\text { Ambos grupos reciben } 2 \text { sesiones de 60'x } \\
\text { semana durante } 15 \text { semanas. }\end{array}$ & $\begin{array}{l}\text { Existe evidencia de efectos positivos } \\
\text { sobre la intensidad del dolor y casi } \\
\text { todos los dominios de la calidad de vida } \\
\text { en pacientes que siguieron Pilates. }\end{array}$ \\
\hline $\begin{array}{l}\text { Priebe et al., } \\
\text { (2013). }\end{array}$ & $\begin{array}{l}\text { Ensayo } \\
\text { controlado } \\
\text { aleatorizado }\end{array}$ & $\begin{array}{l}\text { Pacientes con } \\
\text { esquizofrenia } \\
\mathrm{N}=256 \\
\text { Divididos en gru- } \\
\text { po Psicoterapia y } \\
\text { Pilates }\end{array}$ & $\begin{array}{l}\text { Síntomas negativos(CAINS), psicopato- } \\
\text { logia general (PANSS), contactos sociales } \\
\text { (SIX), depresión (CDSS) y calidad de vida } \\
\text { (MANSA). A partir del manual de Pilates } \\
\text { Union Matwork como guía: } 2 \text { sesiones de } \\
\text { 90’x semana durante } 10 \text { semanas. } \\
\text { Evaluación Pre-post }\end{array}$ & $\begin{array}{l}\text { El grupo Pilates hace de grupo control } \\
\text { a la BPT (psicoterapia corporal). El } \\
\text { BPT llevó a significativamente más ba- } \\
\text { jos niveles de síntomas negativos, tanto } \\
\text { al final del tratamiento y se mantiene a } \\
\text { los } 4 \text { meses de seguimiento. }\end{array}$ \\
\hline \multicolumn{5}{|c|}{$\begin{array}{l}\text { (BDI): Beck Depression Inventory; (CAF): Cuestionario de Autoconcepto Físico; (CAINS): Clinical Assessment Interview for Negative Symptoms; (CDSS): Calgary Depres- } \\
\text { sion Scale; (EQ-5D): European Quality of Life } 5 \text { Dimensions; (ESS): Epworth Sleepiness Scale; (FACT-B): Assessment of Cancer Therapy-Breast; (FDMS): Four Dimensional } \\
\text { Mood Scale; (FFMQ): Five Facet Mindfulness Questionnaire; (FIQ): Fibromyalgia Impact Questionnaire; (GDLAM): Grupo de Desenvolvimiento Latino-Americano para } \\
\text { la Madurez; (GDS): Geriatric Depression Scale; (MANSA): Manchester Short Assessment of Quality of Life; (MBSRQ): Multidimensional Body-Self Relations Questionnai- } \\
\text { re; (NHP): Nottingham Health Profile; (PANSS): Positive and Negative Syndrome Scale; (POMS): Profile of Mood States; (PSQI): Pittsburgh Sleep Quality Index; (QLQ- } \\
\text { C30): Quality of Life Questionnaire; (SBIA): Segmental Bioelectrical Impedance Analysis; (SF-36): Short Form Health Survey; (SIX): Objective Social Outcomes Index; } \\
\text { (SWLS): Satisfaction With Life Scale; (PSS4): Perceived Stress Scale; (SRE): Social Requirements Engineering (WHOQOL-OLD): World Health Organization Quality of } \\
\text { Life Instrument-Older Adults Module. }\end{array}$} \\
\hline
\end{tabular}

Una vez analizados los trabajos que han cumplido con los criterios de inclusión, se observa que la principal variable psicosocial estudiada es la calidad de vida (Altan et al., 2009; Altan et al., 2012; Borges et al., 2013; Gámez-Iruela y Sedeño-Vidal, 2013; González-Gálvez et al., 2012; Küçükçakir et al., 2013; Leopoldino et al., 2013; Priebe et al., 2013; Siqueira et al., 2010; Stan et al., 2012). A ésta le siguen: la depresión (Ekici et al., 2008; Eyigor et al., 2010; Mokhtari et al., 2013; Priebe et al., 2013), el estado de ánimo (Caldwell et al., 2009; Caldwell et al., 2010; Stan et al., 2012), los aspectos relativos al sueño (Caldwell et al., 2009; Caldwell et al., 2010; Leopoldino et tal., 2013) y la autoeficacia generalizada (Caldwell et al., 2009; Caldwell et al., 2010). (Tabla 3).
Tabla 3. Variables psicosociales estudiadas durante la práctica de Pilates

\begin{tabular}{lll}
\hline Variables estudiadas & Número de estudios & Porcentaje \\
\hline Calidad de vida & 9 & 25,00 \\
Depresión & 4 & 11,12 \\
Estado de ánimo & 3 & 8,33 \\
Sueńo & 3 & 8,33 \\
Autoeficacia generalizada & 2 & 5,55 \\
Autoconcepto & 2 & 5,55 \\
Estado de salud & 2 & 5,55 \\
Percepción de funcionalidad & 2 & 5,55 \\
Atención & 1 & 2,78 \\
\hline
\end{tabular}




\begin{tabular}{lll}
\hline Variables estudiadas & Número de estudios & Porcentaje \\
\hline Autonomía & 1 & 2,78 \\
Aprecio por otras personas & 1 & 2,78 \\
Autorregulación & 1 & 2,78 \\
Síntomas negativos & 1 & 2,78 \\
Contactos sociales & 1 & 2,78 \\
Estrés & 1 & 2,78 \\
Psicopatología general & 1 & 2,78 \\
Satisfacción con la vida & 1 & 2,78 \\
Total de variables psicosociales & 36 & 100 \\
\hline
\end{tabular}

La calidad de vida representa una cuarta parte del total de las variables psicosociales estudiadas. Uno de los posibles motivos de este elevado número de investigaciones, sobre la calidad de vida, puede deberse a que el Pilates es muy utilizado dentro del campo de la rehabilitación (González-Gálvez y Sainz de Baranda, 2011). En la presente revisión el 53,33\% de los trabajos de intervención utilizan como muestra a sujetos diagnosticados con alguna enfermedad o dolencia. Teniendo en cuenta que el Método Pilates, en muchas ocasiones, es utilizado desde un punto de vista terapéutico parece comprensible que se evalúe la calidad de vida del practicante. De ahí que muchos investigadores opten por estudiarla en sus trabajos sobre Pilates.

La depresión representa algo más del $11 \%$ del total de variables psicosociales estudiadas, seguida por el estado de ánimo y los aspectos relativos al sueño con algo más del 8\%, cada una. En el caso de la depresión se incluye en tres de los cuatro estudios a: mujeres con cáncer de mama (Eyigor et al., 2010), pacientes con fibromialgia (Ekici et al., 2008) y sujetos diagnosticados de esquizofrenia (Priebe et al., 2013).

El análisis de los aspectos metodológicos empleados en los programas de intervención aportan información valiosa a la hora de desarrollar nuevos estudios. Los trabajos incluidos en la presente revisión han contado con una media de 40,06 sujetos. La frecuencia semanal de práctica de Pilates ha sido de 2.4 días. Respecto a la duración de las clases, los alumnos han asistido a sesiones de 61,25'. También se debe destacar que el estudio de intervención longitudinal más largo emplea un programa de 1 año (Küçükçakir et al., 2013) y el más corto de 4 semanas (Ekici et al., 2008). La duración media de los estudios longitudinales (Segal et al., 2004; Altan et al., 2009; Caldwell et al., 2009; Caldwell et al., 2010; Siqueira et al., 2010; Eyigor et al.,2010; Cruz-Ferreira et al., 2011; Altan et al., 2012; Stan et al., 2012; Leopoldino et al., 2013; Mokhtari et al., 2013; Borges et al., 2013; Priebe et al., 2013) es de 121,33 días. Del mismo modo, las revisiones incluidas aportan datos interesantes sobre el estudio de nuevas variables en relación al método Pilates. Se ha observado una evolución desde la primera revisión de Lange et al. (2000) que se centraba en los beneficios generales del método. Desde entonces las siguientes revisiones se orientaron hacia el análisis del método en adultos sanos (Bernardo et al., 2007; CruzFerreira et al., 2011). Para finalmente, focalizar su atención en pacientes que padecían enfermedades como el cáncer (Stan et al., 2012) o la fibromialgía (Gámez-Iruela y Sedeńo-Vidal, 2013) con el objeto de poder llegar a establecer mejoras en sus niveles de salud.

En relación al apartado laboral, sólo se encuentra un trabajo que relaciona directamente la práctica de Pilates con la salud laboral de un colectivo (Bernardo, 2005). Esta publicación describe los beneficios físicos que pueden obtener las enfermeras y los enfermeros al practicar Pilates. Se considera que las conductas sedentarias favorecen el incremento de peso en la población y por tanto, también, el de los pacientes. Esta nueva situación puede ocasionar lesiones en el cuello, espalda y hombros del colectivo al tener que mover a los enfermos. En definitiva, según el único estudio publicado sobre Pilates y salud laboral, el Método Pilates contribuiría a mejorar su salud laboral, fortaleciendo y tonificando sus músculos.

Tabla 4. Variables laborales encontradas con la palabra clave Pilates en el título, resumen o palabras clave

\begin{tabular}{lll}
\hline Variables laborales estudiadas & Número de estudios & Porcentaje \\
\hline Estrés laboral & 19 & 70,37 \\
Compromiso & 5 & 18,52 \\
Tensión laboral & 2 & 7,41 \\
Satisfacción laboral & 1 & 3,70 \\
Agotamiento & 0 & 0,00 \\
\hline Total de variables laborales & 27 & 100 \\
\hline
\end{tabular}

\section{Discusión}

La falta de bienestar físico, psicológico y/o social de los grupos, en definitiva menor salud, podría generar en pacientes sentimientos negativos y desencadenar posibles signos o síntomas depresivos (Cano, 2005; González, Ortín y Bonillo, 2011). Quizás sea éste uno de los motivos por el que los investigadores estudian la práctica de Pilates como factor de protección ante la depresión. Otro posible motivo de estudio puede estar relacionado con el elevado porcentaje de la población normal que sufre en algún momento de su vida estados de depresión de moderados a intermedios (Márquez, Rodríguez y de Abajo, 2006).

Una posible razón por la que los investigadores relacionan, en sus estudios, Pilates con variables psicológicas como: el estado de ánimo y el estado de salud, la autoeficacia, el autoconcepto, la percepción de funcionalidad, variaciones del sueño, autonomía, síntomas negativos... podría deberse a la popularidad del método dentro del campo de la rehabilitación 
(González-Gálvez y Sainz de Baranda, 2011). Es un sistema de ejercicios muy utilizado entre pacientes que se recuperan de diferentes enfermedades como el cáncer (Eyigor et al., 2010; Stan et al., 2012) o la fibromialgia (Altan et al., 2009; Ekici et al., 2008; Gámez-Iruela y Sedeño-Vidal, 2013). Cuanto más serio es el problema médico, mayor la probabilidad de que la persona sufra trastornos de salud mental (Carter, 2004). Por lo que parece existir una relación directa entre el problema físico, social y/o psicológico que presentan los sujetos y la variable o variables psicosociales elegidas por los investigadores.

Prácticamente todos los autores coinciden en que son necesarias nuevas investigaciones (Altan et al., 2009; Eyigor et al., 2010; Stan et al., 2012) ya que los estudios publicados destacan por la escasa muestra, la falta de un método de Pilates definido y la ausencia de estudios previos en los campos estudiados. Estos aspectos hacen que sea muy difícil encontrar grandes evidencias dentro del apartado psicológico, aquí estudiado.

En general, la dificultad para desarrollar estudios experimentales de causa-efecto, hace que los resultados del ejercicio sobre la salud física estén mejor establecidos que sobre el bienestar psicológico o social (Jiménez, Martínez, Miró y Sánchez, 2008). A pesar de esa dificultad existen evidencias suficientes para apoyar la práctica de ejercicio físico como protector de la salud mental en adultos (Tubic y Dordic, 2013). En el caso de Pilates las dificultades son mayores debido a las singularidades del propio método. No es fácil llevar a cabo estudios longitudinales por la existencia de dos modalidades compatibles de Pilates donde se pueden alternar o combinar clases individuales con clases colectivas. Incluso las agrupaciones suelen ser flexibles por lo que los alumnos pueden cambiar la clase de hora o de día afectando al seguimiento del investigador. Del mismo modo, a la hora de desarrollar un estudio transversal es difícil contar con muestras amplias debido a la propia filosofía del método que otorga mucha importancia a la correcta ejecución de los ejercicios. Esto acaba traduciéndose en que los centros especializados ofrecen sesiones a lo largo del día con muy pocos alumnos en cada una de ellas. Aspecto que dificulta la tarea del investigador a la hora de reclutar un número importante de sujetos.

En el 2010 la Organización Mundial de la Salud (OMS) publica las recomendaciones mundiales sobre actividad física para la salud. La depresión es el único factor de salud mental incluido que cuenta con 4 recomendaciones en adultos de 18 a 64 ańos. La primera es acumular un mínimo de 150 minutos semanales de actividad física aeróbica moderada, o bien un mínimo de 75 minutos de actividad aeróbica vigorosa cada semana, o bien una combinación equivalente de actividad moderada y vigorosa. En el caso del método Pilates, no se puede garantizar que todos los alumnos alcancen esos mínimos marcados por la OMS ya que la metodología más habitual para estudiar Pilates emplea entre 2 y 3 sesiones se- manales de 60'. La segunda es que la actividad aeróbica se realizará en sesiones de 10 minutos, como mínimo. La tercera recomendación es realizar 2 o más veces por semana actividades de fortalecimiento de los grandes grupos musculares que encaja perfectamente en las clases de Pilates. El último criterio aconseja doblar los minutos de actividad física moderada y vigorosa marcados en la primera recomendación si se quieren aumentar los beneficios de la actividad física sobre los niveles de depresión.

A pesar de las dificultades detectadas para poder obtener evidencias sólidas, se va a concretar los beneficios del Método Pilates sobre la salud Psicosocial. Los estudios han demostrado que el Pilates produce efectos significativos en: la depresión de mujeres con fibromialgia (Ekici et al., 2008); la calidad de vida de mujeres con fibromialgia (Altan et al., 2009); el humor en universitarios (Caldwell et al., 2009); el estrés percibido, la atención y el estado de ánimo de jóvenes universitarios (Caldwell et al., 2010); la calidad de vida y la mejora de la autonomía personal de mujeres mayores (Siqueira et al., 2010); la satisfacción con la vida, el estado de salud, la percepción de apreciación de personas, el autoconcepto, la percepción de apariencia física y la funcionalidad de mujeres adultas (Cruz-Ferreira et al., 2011); el estado funcional en pacientes con epicondilitis anquilosante (Altan et al., 2012); la mejora calidad de vida, el estado de ánimo e imagen corporal en mujeres con cáncer de mama (Stan et al., 2012); la calidad de vida en mujeres con osteoporosis (Küçükçakır et al., 2013); la calidad del sueño y la calidad de vida en sedentarios (Leopoldino et al., 2013); la depresión en personas mayores (Mokhtari et al., 2013); la calidad de vida en pacientes con dolor lumbar (Borges et al., 2013). Sin embargo, no todas las intervenciones sobre Pilates han encontrado diferencias estadísticamente significativas para poder asegurar la existencia de efectos beneficiosos sobre la salud. Este es el caso de los trabajos que han evaluado: el estado de salud (Segal et al., 2004), la autoeficacia y la calidad del sueńo entre estudiantes universitarios (Caldwell et al., 2009), la depresión y la calidad de vida en pacientes con cáncer de mama (Eyigor et al., 2010) y los síntomas negativos, psicopatología general, contactos sociales, depresión y calidad de vida en pacientes con esquizofrenia.

Respecto a la salud laboral un único estudio ha evaluado la acción directa del Método Pilates sobre el colectivo sanitario (Bernardo, 2005). Desde nuestro punto de vista, deberían existir más estudios que analizaran el impacto del Método Pilates sobre los factores psicosociales asociados al mundo laboral. Debido a que los riesgos psicosociales, según datos del Instituto Nacional de Seguridad e Higiene en el Trabajo (INSHT), siguen siendo una asignatura pendiente de salud laboral en muchas organizaciones, y por tanto aumenta la exposición de los empleados a estos riesgos.

El Método Pilates tiene una fuerte evidencia para apoyar su uso en la mejora de aspectos físicos como: la flexibilidad, 
equilibrio dinámico, resistencia muscular (Adamy y Loigerot, 2006; Isakowitz, 2009). Sin embargo, tras la revisión de los trabajos analizados se sugiere que en futuras investigaciones se realicen estudios experimentales, longitunales, con mediciones al inicio, a lo largo y al final de la intervención. Es necesario definir el Método de Pilates empleado en cada trabajo así como contar con el mayor número de sujetos posible y que compartan el mismo tipo de Pilates. Sería interesante que se analizaran los beneficios obtenidos al practicar Mat Pilates, máquinas y al hacerlo conjuntamente. Por otra parte, el análisis de nuevas variables psicosociales relacionadas con el trabajo, tales como, estrés laboral, burnout, satisfacción laboral, tensión laboral, engagement, etc., pueden aportar nuevas líneas de estudio acerca del efecto del Método Pilates.

\section{Conclusiones}

El análisis de la literatura realizado muestra que hay una necesidad de definir el tipo de Pilates que se realice para poder entender bien los beneficios psicosociales que pueda aportar esta actividad física. Además, mucha de la evidencia que se tiene es a través de estudios transversales, por lo que sería necesario que los futuros trabajos fueran de corte longitudinal y diferencien los tipos de Pilates empleados. También se considera necesario un abordaje de los efectos de esta práctica sobre las variables psicosociales de los entornos laborales debido a la escasez de publicaciones. En definitiva, se necesitan más trabajos con una mejor metodología para poder dilucidar los efectos que tiene la práctica de Pilates sobre la salud psicosocial de los participantes.

\section{Aplicaciones prácticas}

En el presente estudio se revisa la literatura científica existente que relaciona la práctica de Pilates con la salud psicológica y social. Tras el análisis de las publicaciones incluidas en nuestro trabajo, se observa como el método Pilates es un tipo de actividad física muy enfocada al mantenimiento y la mejora de salud (Anderson y Spector, 2000; Muirhead, 2004). Estos beneficios deberían animar al 42\% de la población española sedentaria (según datos del Eurobarómetro, 2010) a participar del método Pilates o de cualquier otro programa físico-deportivo. Desde nuestro trabajo se remarca la necesidad de ajustar la frecuencia, cantidad y tipo de actividad física realizada a las recomendaciones marcadas en el manual de la OMS sobre actividad física y salud, publicado en el 2010, para llegar a obtener beneficios sobre aspectos psicológicos como la depresión. La importancia que ha alcanzando el método Pilates dentro del campo de la rehabilitación (GonzálezGálvez y Sainz de Baranda, 2011) ha favorecido el aumento de publicaciones que relacionan la práctica del método con el tratamiento y recuperación de enfermedades. Como consecuencia, se abre un nuevo horizonte ante los posibles beneficios que puede reportar la práctica de Pilates entre sujetos que se recuperan de enfermedades como el cáncer de mama (Eyigor et al., 2010) o la fibromialgia (Ekici et al., 2008). La probable aparición de nuevos estudios que se centren en relacionar Pilates con estas y otras patologías puede contribuir a captar el interés de otros campos de investigación como la medicina.

\section{Referencias}

1. Adamany, K. y Loigerot, D. (2006). Pilates: Guía para la mejora del rendimiento. Badalona: Paidotribo.

2. Altan, L., Korkmaz, N., Bingol, Ü. y Gunay, B. (2009). Effect of Pilates Training on People With Fibromyalgia Syndrome: A Pilot Study. Archives of Physical Medicine and Rehabilitation, 90(12), 1983-1988. doi: 10.1016/j.apmr.2009.06.021

3. Altan, L., Korkmaz, N., Dizdar, M. y Yurtkuran, M. (2012). Effect of Pilates training on people with ankylosing spondylitis. Rheumatology International, 32(7), 2093-2099. doi: 10.1007/s00296-011-1932-9

4. Anderson, B. (2005). Randomized clinical trial comparing active versus passive approaches to the treatment of recurrent and chronic low back pain. (Tesis doctoral). University of Miami, Florida.

5. Anderson, B. (2010). Fitting Pilates into a Rehabilitation Practice. The Interdisciplinary Journal of Rehabilitation, 23(5), 24-26.

6. Anderson, B. y Spector, A. (2000). Introduction to Pilates-Based Rehabilitation. Orthopaedic Physical Therapy Clinics of North America, 9(3), 395-410.

7. Bernardo, L.M. (2005). Pilates for nurses. The Pennsylvania Nurse, 60(4), 27.

8. Bernardo, L.M., M.P.H., R.N., H.F.I. (2007). The effectiveness of Pilates training in healthy adults: An appraisal of the research literature. Journal of Bodywork and Movement Therapies, 11, 106-110. doi:10.1016/j. jbmt.2006.08.006
9. Bian, Z., Sun, H., Lu, C., Yao, L., Chen, S. y Li, X. (2013). Effect of Pilates training on alpha rhythm. Computational and Mathematical Methods in Medicine, Article ID 295986. Recuperado de http://dx.doi. org/10.1155/2013/295986

10. Borges, J., Baptista, A.F., Santana, N., Souza, I., Kruschewsky, R.A., Galvão-Castro, B. y Sá, K.N. (2013). Pilates exercises improve low back pain and quality of life in patients with HTLV-1 virus: A randomized crossover clinical trial. Journal of Bodywork and Movement Therapies, 18(1), 68-74. doi: 10.1016/j.jbmt.2013.05.010

11. Caldwell, K., Harrison, M., Adams, M. y Triplett, N.T. (2009). Effect of Pilates and taiji quan training on self-efficacy, sleep quality, mood, and physical performance of college students. Journal of Bodywork and Movement Therapies, 13(2), 155-163. doi: 10.1016/j.jbmt.2007.12.001

12. Caldwell, K., Harrison, M., Adams, M., Quin, R.H. y Greeson, J. (2010). Developing mindfulness in college students through movement-based courses: effects on self-regulatory self-efficacy, mood, stress, and sleep quality. The Journal of American College Health, 58(5), 433442. doi: 10.1080/07448480903540481

13. Cano, A. (2005). Control emocional, estilo represivo de afrontamiento y cáncer: ansiedad y cáncer. Psicooncología, 2(1), 71-80.

14. Carter, R. (2004). La relación entre la salud física y la salud mental: trastornos recurrentes. Norte de Salud Mental, 29, 71-85.

15. Cruz-Ferreira, A., Fernandes, J., Laranjo, L., Bernardo, L.M. y Silva, A. 
(2011). A systematic review of the effects of pilates method of exercise in healthy people. Archives of Physical Medicine and Rehabilitation, 92, 2071-2081. doi: 10.1016/j.apmr.2011.06.018

16. Cruz-Ferreira, A., Fernandes, J., Gomes, D., Bernardo, L.M., Kirkcaldy, B.D., Barbosa, T. y Silva, A. (2011). Effects of pilates-based exercise on life satisfaction, physical self-concept and health status in adult women. Women and Health, 51(3), 240-255. doi: 10.1080/03630242.2011.563417

17. Ekici, G., Yakut, E. y Akbayrak, T. (2008). Effects of Pilates exercises and connective tissue manipulation on pain and depression in females with fibromyalgia: a randomized controlled trial. Journal of Physical Rehabilitation, 19(2), 47-54.

18. Eyigor, S., Karapolat, H., Yesil, H., Uslu, R. y Durmaz, B. (2010). Effects of pilates exercises on functional capacity, flexibility, fatigue, depression and quality of life in female breast cancer patients: a randomized controlled study. European Journal of Physical and Rehabilitation Medicine, 46(4), 481-487.

19. Fernández, E., Santana, F. y Merino, R. (2011). Joseph Hubertus Pilates; anatomía de un gigante olvidado. Trances, 3(3), 353-378.

20. Fernández-Ríos, L. y Buela, G. (2009). Standards for the preparation and writing of Psychology review articles. International Journal of Clinical and Health Psychology, 9(2), 329-344.

21. Gámez-Iruela, J. y Sedeńo-Vidal, A. (2013). Efectividad de la fisioterapia en el abordaje de la fibromialgia. Revisión bibliográfica. Fisioterapia: revista de salud, discapacidad y terapéutica física, 35(5), 224-231.

22. González, J., Ortín, F.J. y Bonillo, J.A. (2011). Actividad física, asistencia psicológica y niveles de ansiedad y depresión en mujeres con fibromialgia: un estudio descriptivo. Cuadernos de Psicología del Deporte, 11(1), 59-66.

23. González-Gálvez, N. y Sainz de Baranda, P. (2011). Aportaciones del Método Pilates desde la Educación Física: Propuesta de progresión. Trances, 3(5), 593-608.

24. González-Gálvez, N., Sainz de Baranda, P., García-Pastor, T. y Aznar, S (2012). Método pilates e investigación: revisión de la literatura. Revista Internacional de Medicina y Ciencias de la Actividad Fisica y el Deporte, 12(48), 771-786.

25. Isakowitz, R. (2009). Pilates: manual completo del método Pilates. Barcelona: Paidotribo.

26. Jiménez, M.G., Martínez, P., Miró, E. y Sánchez, A.I. (2008). Bienestar psicológico y hábitos saludables: ¿están asociados a la práctica de ejercicio físico? International Journal of Clinical and Health Psychology, 8(1), 185-202

27. Keays, K.S., Harris, S.R., Lucyshyn, J.M. y MacIntyre, D.L. (2008). Effects of Pilates exercises on shoulder range of motion, pain, mood, and upper-extremity function in women living with breast cancer: a pilot study. Physical Therapy, 88(4), 494-510. doi: 10.2522/ptj.20070099

28. Küçükçakır, N., Altan, L. y Korkmaz, N. (2013). Effects of Pilates exercises on pain, functional status and quality of life in women with postmenopausal osteoporosis. Journal of bodywork and movement therapies, 17(2), 204-211. doi: 10.1016/j.jbmt.2012.07.003

29. Lange, C., Unnithan, V.B., Larkam, E. y Latta, P.M. (2000). Maximizing the benefits of Pilates-inspired exercise for learning functional motor skills. Journal of Bodywork and Movement Therapies, 4(2), 99-108.

30. Latey, P. (2001). The Pilates method: history and philosophy. Journal of Bodywork and Movement Therapies, 5(4), 275-282.

31. Leopoldino, A.A., Avelar, N.C., Passos, G.B., Jr Santana, N.A., Jr Teixeira, V.P., Jr de Lima, V.P. y de Melo Vitorino, D.F. (2013). Effect of Pilates on sleep quality and quality of life of sedentary population. Journal of Bodywork and Movement Therapies, 17(1), 15-10. doi: 10.1016/j. jbmt.2012.10.001

32. Levine, B., Kaplanek, B., Scafura, D. y Jaffe, W.L. (2007). Rehabilitation after total hip and knee arthroplasty: a new regimen using Pilates training. Bulletin New York University Hospital for Joint Diseases, 65(2), 120-125.
33. López Menchero, J.L. (2009). La salud laboral de los docentes. Recuperado de http://www.educaweb.com/noticia/2009/03/26/salud-laboraldocentes-13542.html

34. Márquez, S., Rodríguez, J. y de Abajo, S. (2006). Sedentarismo y salud: efectos beneficiosos de la actividad física. Apunts de Educación física y deportes, 83, 12-24.

35. McGrath, J.A., O’Malley, M. y Hendrix, T.J. (2011). Group exercise mode and health-related quality of life among healthy adults. Journal of Advanced Nursing, 67(3), 491-500. doi: 10.1111/j.13652648.2010.05456.x

36. Mendonça, T.M., Terreri, M.T., Silva, C.H., Neto, M.B., Pinto, R.M., Natour, J. y Len, C.A. (2013). Effects of Pilates Exercises on HealthRelated Quality of Life in Individuals with Juvenile Idiopathic Arthritis. Archives of Physical Medicine and Rehabilitation, 94(11), 2093 3102. doi: 10.1016/j.apmr.2013.05.026

37. Mokhtari, M., Nezakatalhossaini, M. y Esfarjani, F. (2013). The Effect of 12 Week Pilates Exercises on Depression and Balance Associated with Falling in the Elderly. Procedia-Social and Behavioral Sciences, 70, 1714-1723. doi: 10.1016/j.sbspro.2013.01.246

38. Muirhead, M. (2004). Total Pilates. Madrid: Pearson.

39. Perestelo, L. (2013). Standards on how to develop and report systematic reviews in Psychology and Health. International Journal of Clinical and Health Psychology, 13, 49-57. Recuperado de http://dx.doi.org/10.1016/ S1697-2600(13)70007-3

40. Pilates, J. y Miller, W. (1945). Pilates Return to life Through Contrology. Incline Village, Nevada: Presentation Dynamics Incorporated.

41. Priebe, S., Savill, M., Reininghaus, U., Wykes, T., Bentall, R., Lauber, C., McCrone, P., Röhricht, F. y Eldridge, S. (2013). Effectiveness and cost-effectiveness of body psychotherapy in the treatment of negative symptoms of schizophrenia a multi-centre randomised controlled trial. BioMedical Central Psychiatry, 13(26), 1-8. doi: 10.1186/1471244X-13-26.

42. Ramos, D. (2007). Método Pilates: las lecciones de las empresas que dominan un sector de gran crecimiento. Emprendedores: las claves de la economía y el éxito profesional, 117, 30-34.

43. Sánchez-Meca, J. y Botella, J. (2010). Revisiones sistemáticas y metaanálisis: Herramientas para la práctica profesional. Papeles del Psicólogo, 31(1), 7-17.

44. Segal, N.A., Hein, J. y Basford, J.R. (2004). The effects of Pilates training on flexibility and body composition: an observational study. Archives of Physical Medicine and Rehabilitation, 85(12), 1977-1981.

45. Serrano, M.A. y Boix, S. (2012). Efectos del tipo y cantidad de actividad física en la salud psicológica percibida de profesoras. Revista Iberoamericana de Psicología del Ejercicio y el Deporte, 7(1), 149-161.

46. Shedden, M. y Kravitz, L. (2006). Pilates Exercise. A Research-Based Review. Journal of Dance Medicine of Science, 10(3), 111-116.

47. Siler, B. (2000). The Pilates Body. New York: Broadway Books.

48. Siqueira, B.G., Cader, S.A., Oliver, N.V., Monteiro de Oliveira, E. y Martin, E.H. (2010). Pilates method in personal autonomy, static balance and quality of life of elderly females. Journal of Bodywork and Movement Therapies, 14(2), 195-202. doi: 10.1016/j.jbmt.2009.12.005

49. Stan, D.L, Collins, N.M., Olsen, M.M., Croghan, I. y Pruthi, S. (2012). The evolution of mindfulness-based physical interventions in breast cancer survivors. Evidence Based Complementary and Alternative Medicine. doi:10.1155/2012/758641

50. Stan, D.L., Rausch, S.M., Sundt, K., Cheville, A.L., Youdas, J.W., Krause, D.A., Boughey, J.C., Walsh, M.F., Cha, S.S. y Pruthi, S. (2012). Pilates for breast cancer survivors. Clinical Journal of Oncology Nursing, 16(2), 131-141.doi: 10.1188/12.CJON.131-141

51. Tinoco, M. y Jiménez, M. (2010). Revisión bibliográfica de los estudios de investigación relacionados con el Método Pilates. Scientia, 15(2), 105-124.

52. Tubic, T. y Dordic, V. (2013). Exercise effects on mental health of pres- 
chool children. Anales de Psicología, 29 (1), 249-256. doi: http://dx.doi. org/10.6018/analesps. 29.1.130721

53. Vieira, F.T., Faria, L.M., Wittmann, J.I., Teixeira, W. y Nogueira, L.A. (2013). The influence of Pilates method in quality of life of practitioners. Journal of Bodywork and Movement Therapies, 17(4), 483-487. doi: 10.1016/j.jbmt.2013.03.006
54. Warr, P.B. (2003). Well-being and the workplace, en Kahneman, D., Diener, E. y Schwarz, N. (Eds): Well-being. The foundations of hedonic psychology (392-412). New York: Russell Sage Foundation.

55. Winsor, M. (2002). Pilates: el centro de energía. Barcelona: Paidotribo. 
